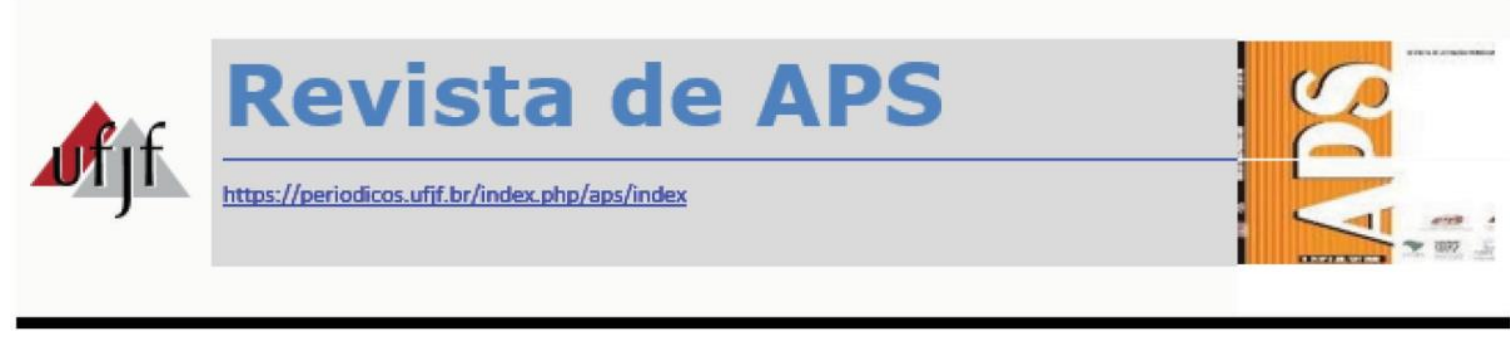

\title{
O tratamento da tuberculose sob a ótica dos profissionais da Atenção Primária à Saúde
}

\section{Tuberculosis treatment under the optics of Primary Health Care professionals}

\author{
Tatiane Cabral Siqueira ${ }^{1}$, Rafaele Oliveira Bonfim² ${ }^{2}$, Melisane Regina Lima Ferreira ${ }^{3}$, \\ Valéria Moreira da Silva ${ }^{4}$, Nathália Halax Orfão ${ }^{5}$
}

\begin{abstract}
RESUMO
O acesso à saúde, em especial, a Atenção Primária à Saúde (APS), é fundamental para o fortalecimento do Sistema Único de Saúde (SUS). Enquanto problema de saúde pública, o controle da tuberculose (TB) depende desse aspecto, organizacional e de desempenho, que se refere à primeira etapa do percurso realizado pelo usuário quando este busca pela satisfação de uma necessidade de saúde. Em vista disso, objetivou-se analisar as ações e estratégias para o tratamento da TB na APS no município de Porto Velho-RO, sob a ótica dos profissionais de saúde. Estudo descritivo, do tipo inquérito, realizado de forma transversal a partir da abordagem quantitativa. A coleta de dados foi realizada por meio das dimensões acesso ao tratamento e formação profissional da versão para profissionais de saúde do formulário "Primary Care Assessment Tool" (PCATool), validado para o Brasil e adaptado para TB, cuja categoria de resposta varia segundo a escala Likert. Os dados foram analisados por meio de estatística descritiva, após aprovação do Comitê de Ética em Pesquisa, conforme parecer 2.585.934 CEP/UNIR. Responderam ao formulário 266 profissionais, sendo 119 Agentes Comunitários de Saúde (ACS), 77 técnicos/auxiliares de enfermagem, 43 enfermeiros e 27 médicos. A dimensão acesso ao tratamento obteve escore geral igual a 3,15 (às vezes) e a dimensão

\footnotetext{
${ }^{1}$ Enfermeira. Especializanda em Saúde Pública na Fundação Universidade Federal de Rondônia (UNIR). E-mail: tatianecabralsiqueira@gmail.com

2 Enfermeira. Mestranda no Programa de Pós-Graduação Enfermagem em Saúde Pública da Escola de Enfermagem de Ribeirão Preto (EERP), Universidade de São Paulo, Ribeirão Preto (SP).

${ }^{3}$ Enfermeira. Especializanda em Saúde Pública na Fundação Universidade Federal de Rondônia (UNIR).

${ }^{4}$ Enfermeira. Mestre em Ensino em Ciências da Saúde. Docente do Departamento de Enfermagem da Universidade Federal de Rondônia (UNIR).

${ }^{5}$ Enfermeira. Doutora em Enfermagem em Saúde Pública. Docente do Departamento de Enfermagem da Universidade Federal de Rondônia (UNIR).
} 
formação profissional escore geral igual a 3,99 (quase sempre). Verificou-se um desconhecimento quanto às barreiras de acesso enfrentadas pelos usuários, bem como divergências entre as categorias profissionais quanto aos atributos da APS, o que evidencia a fragmentação da assistência. Nesse sentindo, é essencial ampliar a capacitação a todos os membros da equipe de saúde e associá-la ao contexto social da comunidade.

PALAVRAS-CHAVE: Profissional de Saúde. Terapêutica. Tuberculose.

\begin{abstract}
The access to health, in particular, to the Primary Health Care (PHC), is fundamental for the strengthening of the Unified Health System (UHS). As a public health problem, tuberculosis (TB) control depends on this organizational aspect and performance, which refers to the first stage of the path taken by the user when he seeks to satisfy a health need. In view of this, the objective was to analyze the actions and strategies for the treatment of TB in PHC in the city of Porto Velho-RO, from the perspective of health professionals. This descriptive, survey-type study, was crosssectionally carried out using the quantitative approach. Data collection was carried out through the dimensions of access to treatment and professional training of the form "Primary Care Assessment Tool" (PCATool) in the version for health professionals, validated for Brazil and adapted for TB, whose response category varies according to the Likert scale. The data were analyzed using descriptive statistics, after approval by the Research Ethics Committee, according to opinion 2,585,934 CEP/UNIR. 266 professionals responded to the form, 119 of whom were Community Health Agents (CHA), 77 nursing technicians/assistants, 43 nurses and 27 doctors. The dimension of access to treatment obtained a general score of 3.15 (sometimes), and the dimension of professional training a general score of 3.99 (almost always). There was a lack of knowledge regarding the barriers of access faced by users, as well as differences between the professional categories regarding the attributes of PHC, which shows the fragmentation of care. In this sense, it is essential to expand training to all members of the health team and associate it with the social context of the community.
\end{abstract}

KEYWORDS: Health Professional. Therapeutics. Tuberculosis.

\title{
INTRODUÇÃO
}

O acesso à saúde, em especial, a Atenção Primária à Saúde (APS), é fundamental para o fortalecimento do Sistema Único de Saúde (SUS), que tem como princípios doutrinários a universalidade, a integralidade e a equidade. Contudo, ainda persistem desafios para garantir o acesso universal e equânime dos serviços de saúde à população brasileira, tendo em vista as transformações demográficas, epidemiológicas e as desigualdades sociais que caracterizam o cenário nacional ${ }^{1}$.

$O$ acesso é um aspecto organizacional e de desempenho que se refere à primeira etapa do percurso realizado pelo usuário quando este busca pela satisfação de uma necessidade de saúde ${ }^{2}$. Conceitualmente, divide-se em geográfico, organizacional 
e social ${ }^{3}$, os quais estão relacionados à distância entre a população e os recursos dos serviços de saúde, ao uso de transporte e ao tempo gasto para obtenção de assistência; às características e recursos que facilitam ou dificultam os cuidados em saúde; e aos aspectos socioeconômicos vinculados ao indivíduo 4 .

Para as condições crônicas, tais aspectos podem acarretar dificuldades no acesso aos serviços de saúde e retardo no diagnóstico e tratamento de doenças como a tuberculose (TB), a qual tem suas ações de controle descentralizada para APS, conforme preconizado pelo Ministério da Saúde (MS).

O Brasil é prioritário para o enfrentamento da TB e da coinfecção com o vírus da imunodeficiência humana (HIV), por ser um dos 30 países que compõem as listas de alta carga destas morbidades ${ }^{5}$. Em 2017, apresentou 69.569 casos novos da doença e 4.534 óbitos, além da baixa taxa de cura $(71,4 \%)$ e elevada taxa de abandono $(10,8 \%)^{6,7}$, quando comparado às metas da OMS de no mínimo $85 \%$ e no máximo $5 \%$, respectivamente ${ }^{8}$.

Porto Velho, uma das capitais com maior proporção de retratamentos da doença (23,3\%), apresentou, em 2017, 59,1 e 2,5 casos de incidência e mortalidade de TB a cada 100 mil habitantes, respectivamente, além de indicadores operacionais aquém do preconizado pela OMS, sendo a cura de $67,4 \%$ e o abandono de $18,6 \%$.

Esse cenário é agravado pelo fato de mais da metade dos casos de a doença no município serem diagnosticados e notificados no serviço de referência terciária ${ }^{9}$, apesar da responsabilidade da APS por todas as ações que permeiam o manejo da TB, sendo os demais níveis de atenção destinados a ações específicas, quando necessário.

Nesse contexto, tendo em vista que avanços dependem de melhorias no diagnóstico e tratamento da doença, infecção latente da TB (ILTB) e determinantes sociais de saúde ${ }^{10}$, barreiras de acesso ao tratamento são preditoras para o abandono. Um dos principais desafios para o controle da doença, considerando que tal desfecho leva à manutenção da cadeia de transmissão, aumento da incidência, resistência bacteriana e óbito 5 .

Com objetivo de garantir a adesão a terapia, o Brasil aderiu à estratégia Directly Observed Treatment ShortCourse (DOTS), que tem como principal pilar o Tratamento Diretamente Observado (TDO), no qual ocorre a observação direta da ingesta medicamentosa. Contudo, a dificuldade de acesso e organização dos serviços de saúde, bem como a não corresponsabilização dos profissionais para deteç̧ão precoce dos casos, influenciaa diretamente no retardo do diagnóstico e início do tratamento, o que contribui com desfechos desfavoráveis ${ }^{11}$.

Observa-se consonância entre perfil sociodemográfico e clínico dos doentes que abandonam o tratamento e foram a óbito pela doença em Porto Velho com a literatura ${ }^{11,12}$. Destes, a maioria dos casos que tiveram como desfecho o óbito não foram acompanhados pelo TDO ${ }^{12}$. 
Esses fatores estão relacionados às condições sociais, econômicas, clínicas, estruturais e organizacionais dos serviços de saúde ${ }^{13,14}$. Portanto, avaliar como vem acontecendo o acesso da população ao tratamento da TB pode viabilizar mudanças no cenário atual, por meio da identificação das fragilidades da APS em garantir o tratamento, acompanhamento e monitoramento dos casos, para que se possa buscar o controle da doença enquanto problema de saúde pública.

Assim, objetivou-se analisar as ações e estratégias para o tratamento da tuberculose na Atenção Primária à Saúde no município de Porto Velho-RO, sob a ótica dos profissionais de saúde.

\section{MATERIAL E MÉTODOS}

Trata-se de um estudo descritivo, do tipo inquérito, realizado de forma transversal a partir da abordagem quantitativa, desenvolvido em Porto Velho, capital do estado de Rondônia. O estado possui a atenção à TB descentralizada para APS, considerada porta de entrada e o primeiro contato para os sintomáticos respiratórios (SR) 5 .

A APS ainda é responsável pela avaliação clínica, solicitação dos exames diagnósticos, manejo do cuidado - incluindo acompanhamento e controle mensal - TODO, avaliação dos contatos para investigação da infecção latente da TB (ILTB) e encaminhamento do doente aos serviços de referência, quando necessário.

Para a organização desse fluxo, o município apresenta 19 unidades de saúde na área urbana, sendo 17 Unidades de Saúde da Família (USF) e 2 Unidades Básicas de Saúde (UBS). Essas unidades contam com 60 equipes de saúde ${ }^{15}$, com cobertura de $46,15 \%$ da população em $2019^{16}$.

A população do estudo foi constituída pelos profissionais que atuam na APS da zona urbana do município de Porto Velho-RO. Como critérios de inclusão, foram considerados os profissionais que já acompanharam pelo menos um caso de TB e que exercem sua função há pelo menos 12 meses na unidade em que foram entrevistados. E como critérios de exclusão, aqueles que estavam de licença e/ou férias durante o período da coleta de dados.

Tendo em vista a população de 737 profissionais nessas unidades ${ }^{15}$, o n amostral foi estimado pela fórmula da população finita:

$$
n=\frac{z_{\frac{\alpha}{2}}^{2} * N * P *(1-P)}{\varepsilon^{2} *(N-1)+z_{\frac{\alpha}{2}}^{2} *(1-P)}
$$

Nesse sentido, considerando um erro $(\varepsilon)$ de $5 \%$, um intervalo de confiança $(Z)$ de $95 \%$ e uma proporção amostral (P) de $50 \%$ da população (N), deveriam ser entrevistados, no mínimo, 253 profissionais, sendo 136 ACS, 65 técnicos/auxiliares de enfermagem, 26 enfermeiros e 26 médicos. 
Os dados foram coletados entre maio de 2018 e fevereiro de 2019 a partir do formulário "Primary Care Assessment Tool" (PCATool)2, validado para o Brasil ${ }^{17}$ e adaptado para TB em três versões: doentes de TB, profissionais de saúde e gestores ${ }^{18}$.

Para atender o objetivo deste estudo, foi considerada apenas a versão para profissionais de saúde, que contém 97 questões, divididas em 10 dimensões: informações gerais, porta de entrada, acesso ao diagnóstico, acesso ao tratamento, vínculo, elenco de serviços, coordenação, enfoque na família, orientação para a comunidade e formação profissional.

Com o intuito de responder aos objetivos propostos, foram selecionadas para este estudo as dimensões "acesso ao tratamento" e "formação profissional" (Quadro 1). Essas são representadas no formulário pelas letras " $D$ " e "J", respectivamente. Por esse motivo, foram mantidas essas representações nas figuras, associadas aos números representativos de cada variável. A primeira dimensão contempla 11 variáveis, numeradas de 1 a 11; e a segunda, com oito variáveis, numeradas de 1 a 8 .

Quadro 1-Dimensões e variáveis selecionadas para o estudo

\begin{tabular}{|c|c|c|}
\hline DIMENSÃO & \multicolumn{2}{|l|}{ VARIÁVEIS } \\
\hline \multirow{11}{*}{$\begin{array}{l}\text { ACESSO AO } \\
\text { TRATAMENTO }\end{array}$} & $\begin{array}{l}\text { Consulta médica no prazo de } 24 \text { horas, se os doentes em tratamento } \\
\text { passarem mal por causa da medicação ou da TB }\end{array}$ & D1 \\
\hline & $\begin{array}{l}\text { Obtenção de informações durante o tratamento por telefone na } \\
\text { unidade de saúde }\end{array}$ & D2 \\
\hline & $\begin{array}{l}\text { Marcação de consulta durante o tratamento por telefone na } \\
\text { unidade de saúde }\end{array}$ & D3 \\
\hline & Visitas domiciliares aos doentes de TB pelos profissionais & D4 \\
\hline & $\begin{array}{l}\text { Frequência de pertencimento à área adscrita pelos doentes de TB } \\
\text { tratados na unidade }\end{array}$ & D5 \\
\hline & $\begin{array}{l}\text { Perda de turno de trabalho ou compromisso durante o tratamento } \\
\text { da TB para serem atendidos }\end{array}$ & D6 \\
\hline & $\begin{array}{l}\text { Dificuldade pelos usuários para se deslocar até a unidade de saúde } \\
\text { onde fazem tratamento para serem consultados }\end{array}$ & D7 \\
\hline & $\begin{array}{l}\text { Utilização de algum tipo de transporte motorizado pelos doentes } \\
\text { de TB para se deslocar até a unidade de saúde }\end{array}$ & D8 \\
\hline & Pagamento pelo transporte pelos doentes de TB & D9 \\
\hline & Falta de medicamentos para TB durante os últimos 12 meses & D10 \\
\hline & $\begin{array}{l}\text { Espera dos doentes de TB para serem atendidos por mais de } 60 \\
\text { minutos }\end{array}$ & D11 \\
\hline
\end{tabular}


(Conclusão)

\begin{tabular}{|c|c|c|}
\hline DIMENSÃO & \multicolumn{2}{|l|}{ VARIÁVEIS } \\
\hline \multirow{8}{*}{$\begin{array}{l}\text { FORMAÇÃO } \\
\text { PROFISSIONAL }\end{array}$} & Tempo de atuação nas ações de controle da TB & J1 \\
\hline & Abordagem do tema TB durante a formação & $\mathrm{J} 2$ \\
\hline & $\begin{array}{l}\text { Vivência da temática da TB em disciplinas ou estágios durante a } \\
\text { formação }\end{array}$ & J3 \\
\hline & $\begin{array}{l}\text { Recebimento de treinamento específico no serviço para atuação } \\
\text { em ações de controle da TB }\end{array}$ & J4 \\
\hline & Qualificação dos profissionais para o atendimento de TB & J5 \\
\hline & $\begin{array}{l}\text { Qualificação dos profissionais para atuação segundo a diversidade } \\
\text { cultural da comunidade }\end{array}$ & J6 \\
\hline & $\begin{array}{l}\text { Realização de educação permanente para o desenvolvimento de } \\
\text { ações de controle da TB }\end{array}$ & $\mathrm{J} 7$ \\
\hline & $\begin{array}{l}\text { Seguimento de algum protocolo específico para as ações de } \\
\text { controle da TB na unidade de saúde }\end{array}$ & J8 \\
\hline
\end{tabular}

Fonte: elaborado pelas autoras

Em cada pergunta, os profissionais tiveram cinco possibilidades de resposta, segundo a escala Likert: nunca, quase nunca, às vezes, quase sempre ou sempre, as quais são classificadas por meio de uma variação de um a cinco, sendo 1 o pior desempenho e 5 o melhor desempenho ${ }^{18}$.

Os dados coletados foram armazenados no Programa Microsoft Excel e analisados por meio de estatística descritiva no software Statistic 13.0, da Statsoft. Determinou-se um escore médio para cada variável, que corresponde à somatória de todas as categorias de respostas dividida pelo total de participantes. De modo complementar, foi definido o escore médio da dimensão elencada para este estudo, que corresponde à somatória de todos os escores médios das variáveis dividida pelo total de variáveis ${ }^{18}$.

Conforme a escala Likert de resposta do instrumento, adotaram-se os seguintes parâmetros para categorizar os escores médios obtidos: nunca $(1,00-1,50)$, quase nunca $(1,51-2,50)$, às vezes $(2,51-3,50)$, quase sempre $(3,51-4,50)$ e sempre $(4,51-5,00)$.

Atendendo às recomendações da resolução 466/12 do Conselho Nacional de Saúde ${ }^{19}$, o projeto matriz intitulado "Dimensões organizacionais e de desempenho dos serviços de saúde para o manejo do cuidado aos doentes de tuberculose em Porto Velho-RO" foi aprovado pelo Comitê de Ética em Pesquisa (CEP) da Fundação Universidade Federal de Rondônia (UNIR), conforme número de parecer 2.585.934 CEP/UNIR.

As entrevistas ocorreram após autorização da SEMUSA e assinatura do Termo de Consentimento Livre e Esclarecido (TCLE) pelo profissional, na unidade de saúde em que atuava e em local que resguardasse a privacidade do mesmo. 


\section{RESULTADOS}

Ainda que 737 profissionais estejam cadastrados no Cadastro Nacional de Estabelecimentos de Saúde (CNES), verificou-se que apenas 688 eram atuantes nas 19 unidades de saúde do município, cujo quantitativo por profissão é de 362 ACS, 172 técnicos/auxiliares de enfermagem, 74 enfermeiros e 80 médicos. Destes, 401 foram abordados durante a coleta de dados, entretanto 120 foram excluídos (65 ACS, 33 técnicos/auxiliares de enfermagem, 13 enfermeiros e 9 médicos), sendo 73 por nunca terem acompanhado caso de TB, 29 por férias, 14 por licença e 4 por atuarem há menos de 12 meses na unidade.

Outros 15 profissionais (6ACS, 4 técnicos/auxiliares de enfermagem, 2 enfermeiros e 3 médicos) se recusaram a participar, por não possuírem interesse na pesquisa, quererem responder o formulário de uma forma diferente da proposta metodológica, por falta de disponibilidade ou não ter obtido feedback de pesquisas anteriormente. Assim, 266 foram entrevistados (119 ACS, 77 técnicos/auxiliares de enfermagem, 43 enfermeiros e 27 médicos), sendo 245 de Equipe de Saúde da Família (eSF) e 21 de Equipe de Atenção Básica (eAB). Esses apresentaram média na função de 10,89 anos (dp $= \pm 5,55$ anos), sendo o mínimo de um e máximo de 38 anos.

Quanto à dimensão acesso ao tratamento (média do escore geral = 3,15 - às vezes), os profissionais afirmaram que o doente em tratamento sempre consegue consulta médica no prazo de 24 horas, se passar mal por causa da medicação ou da TB. Quase sempre esses profissionais realizam visitas domiciliares aos doentes e estes pertencem à área adscrita à unidade de saúde onde são tratados. De modo complementar, às vezes os doentes perdem turno de trabalho ou compromisso para serem atendidos durante o tratamento e têm que utilizar algum tipo de transporte motorizado para se deslocar até a unidade de saúde, bem como pagar pelo mesmo (Figura 1).

Também acerca dessa dimensão, os profissionais responderam que quase nunca os doentes de TB conseguem obter informações por telefone na unidade de saúde, têm dificuldade para se deslocar até o serviço onde fazem tratamento para serem consultados, faltaram medicamentos para TB durante os últimos 12 meses e os doentes de TB esperam mais de 60 minutos para serem atendidos, bem como nunca conseguem marcar consulta por telefone na unidade de saúde (Figura 1). 
Figura 1 - Representação gráfica da média e do intervalo de confiança dos escores da dimensão acesso ao tratamento, obtidos pelos profissionais entrevistados da APS em

Porto Velho-RO, no período de maio de 2018 a fevereiro de 2019

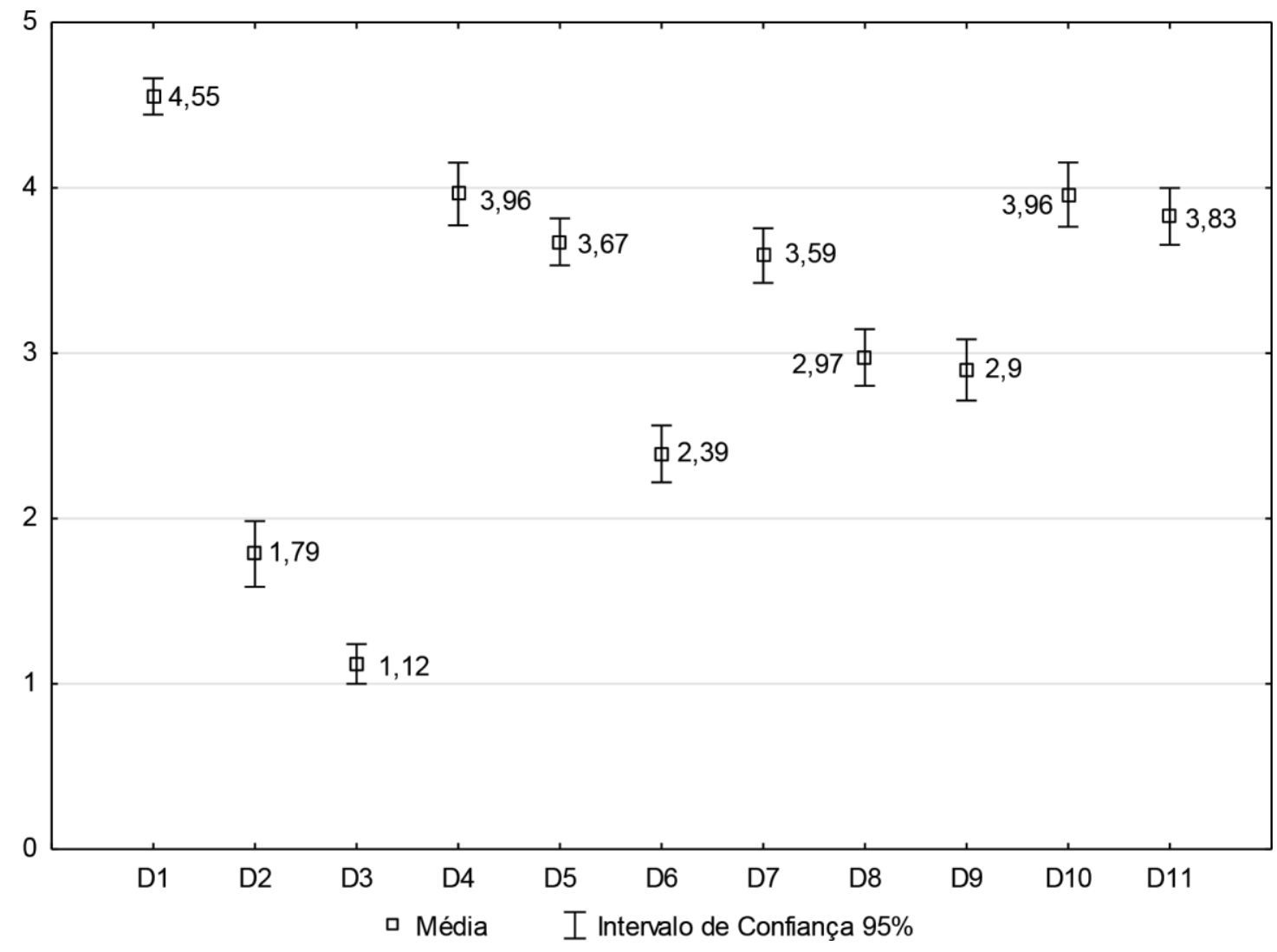

Legenda:

D1 - consulta médica no prazo de 24 horas, se os doentes em tratamento passarem mal por causa da medicação ou da TB; D2 - obtenção de informações durante o tratamento por telefone na unidade de saúde; D3 - marcação de consulta durante o tratamento por telefone na unidade de saúde; D4 - visitas domiciliares aos doentes de TB pelos profissionais; D5 - frequência de pertencimento à área adscrita pelos doentes de TB tratados na unidade; D6 - perda de turno de trabalho ou compromisso durante o tratamento da TB para serem atendidos; D7 - dificuldade pelos usuários para se deslocar até a unidade de saúde onde fazem tratamento para serem consultados; D8 - utilização de algum tipo de transporte motorizado pelos doentes de TB para se deslocar até a unidade de saúde; D9 - pagamento pelo transporte pelos doentes de TB; D10 - falta de medicamentos para TB durante os últimos 12 meses; D11 - espera dos doentes de TB para serem atendidos por mais de 60 minutos

Fonte: elaborado pelas autoras

De acordo com a categoria profissional, verificou-se classificação superior (sempre) entre ACS e similaridade com classificação inferior (às vezes) entre médicos e enfermeiros sobre a frequência das visitas domiciliares aos doentes de TB pelos profissionais quando comparados com técnicos/auxiliares de enfermagem. Em relação ao pertencimento à área adscrita pelos doentes de TB tratados na unidade, médicos e enfermeiros também apresentaram classificação inferior (às vezes) quando comparados 
com técnicos/auxiliares de enfermagem e ACS. De modo complementar, a equipe de enfermagem e os ACS divergem dos médicos quanto à perda de turno de trabalho ou compromisso durante o tratamento da doença para serem atendidos (Figura 2).

Sobre a dificuldade pelos usuários para se deslocar até a unidade de saúde onde fazem tratamento para serem consultados, médicos e ACS relataram que isso ocorre quase sempre, enquanto que a equipe de enfermagem tem a percepção de que ocorre às vezes. Enfermeiros consideraram em menor proporção (quase nunca) a necessidade do doente de pagamento pelo transporte até a unidade de saúde, divergindo, assim, dos demais profissionais (Figura 2).

Figura 2 - Representação gráfica da média e do intervalo de confiança dos escores da dimensão acesso ao tratamento, obtidos pelos profissionais enfermeiros (a), médicos (b), técnicos/auxiliares de enfermagem (c) e Agentes Comunitários de Saúde (d) entrevistados da APS em Porto Velho-RO, no período de maio de 2018 a fevereiro de 2019

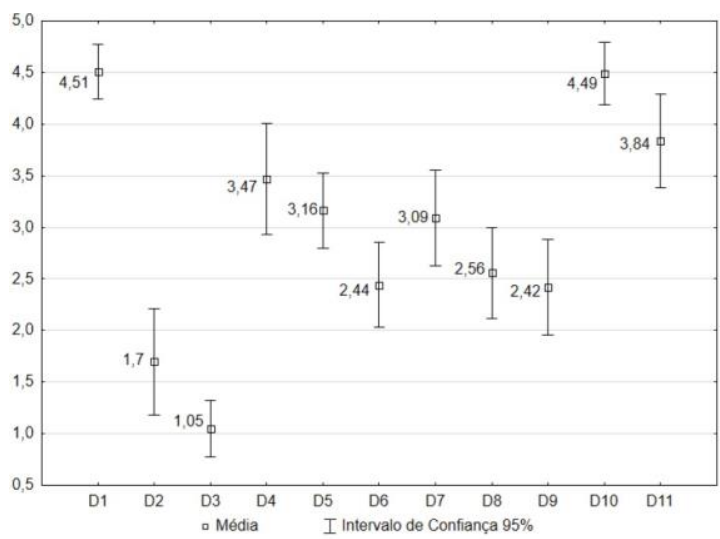

(a)

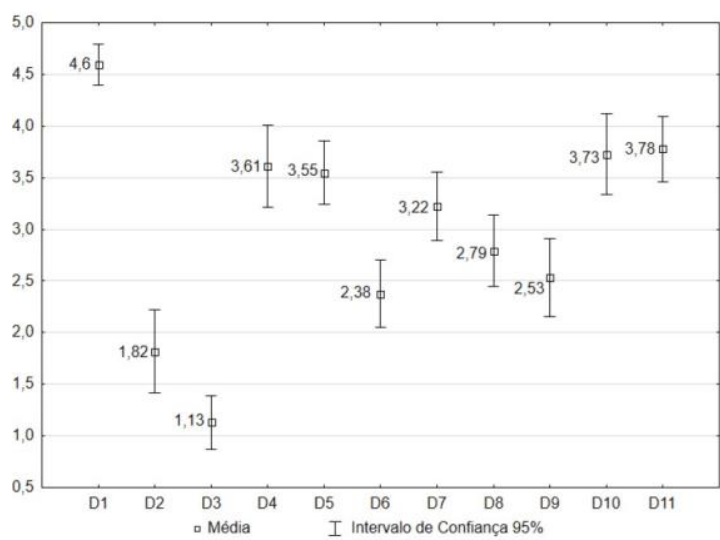

(c)

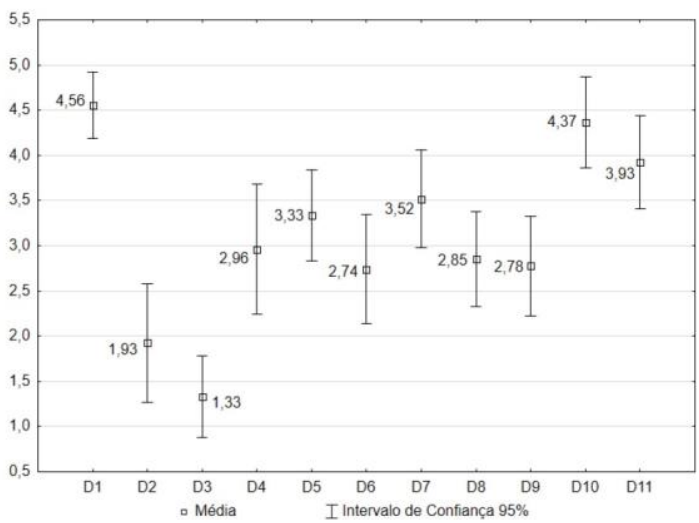

(b)

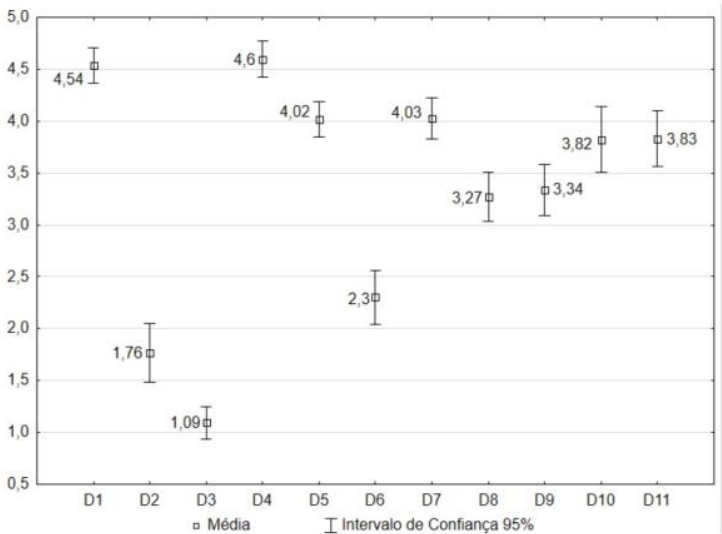

(d)

Legenda: D1 - consulta médica no prazo de 24 horas, se os doentes em tratamento passarem mal por causa da medicação ou da TB; D2 - obtenção de informações durante o tratamento por telefone na unidade de saúde; D3 - marcação de consulta durante o tratamento por telefone na unidade de saúde; D4 - visitas domiciliares aos doentes de TB pelos profissionais; D5 - frequência de pertencimento à área adscrita pelos doentes de TB tratados na unidade; D6 - perda de turno de trabalho ou compromisso durante o 
tratamento da TB para serem atendidos; D7 - dificuldade pelos usuários para se deslocar até a unidade de saúde onde fazem tratamento para serem consultados; D8 - utilização de algum tipo de transporte motorizado pelos doentes de TB para se deslocar até a unidade de saúde; D9 - pagamento pelo transporte pelos doentes de TB; D10 - falta de medicamentos para TB durante os últimos 12 meses; D11 - espera dos doentes de TB para serem atendidos por mais de 60 minutos

Fonte: elaborado pelas autoras

$\mathrm{Na}$ dimensão formação profissional (média do escore geral = 3,99 - quase sempre), identificou-se que sempre os trabalhadores atuam em ações de controle da TB há quatro anos ou mais e durante a sua formação o tema foi abordado. Quase sempre houve disciplinas ou estágios que permitiram vivenciar a temática, como também receberam treinamento específico no serviço para atuarem em ações de controle da doença, são qualificados para atender TB e trabalhar segundo a diversidade cultural da comunidade e seguem algum protocolo específico para as ações de controle da doença na unidade de saúde. Porém, somente às vezes realizam educação permanente para desenvolver essas atividades (Figura 3).

Figura 3 - Representação gráfica da média e do intervalo de confiança dos escores da dimensão formação profissional, obtidos pelos profissionais da APS entrevistados em

Porto Velho-RO, no período de maio de 2018 a fevereiro de 2019

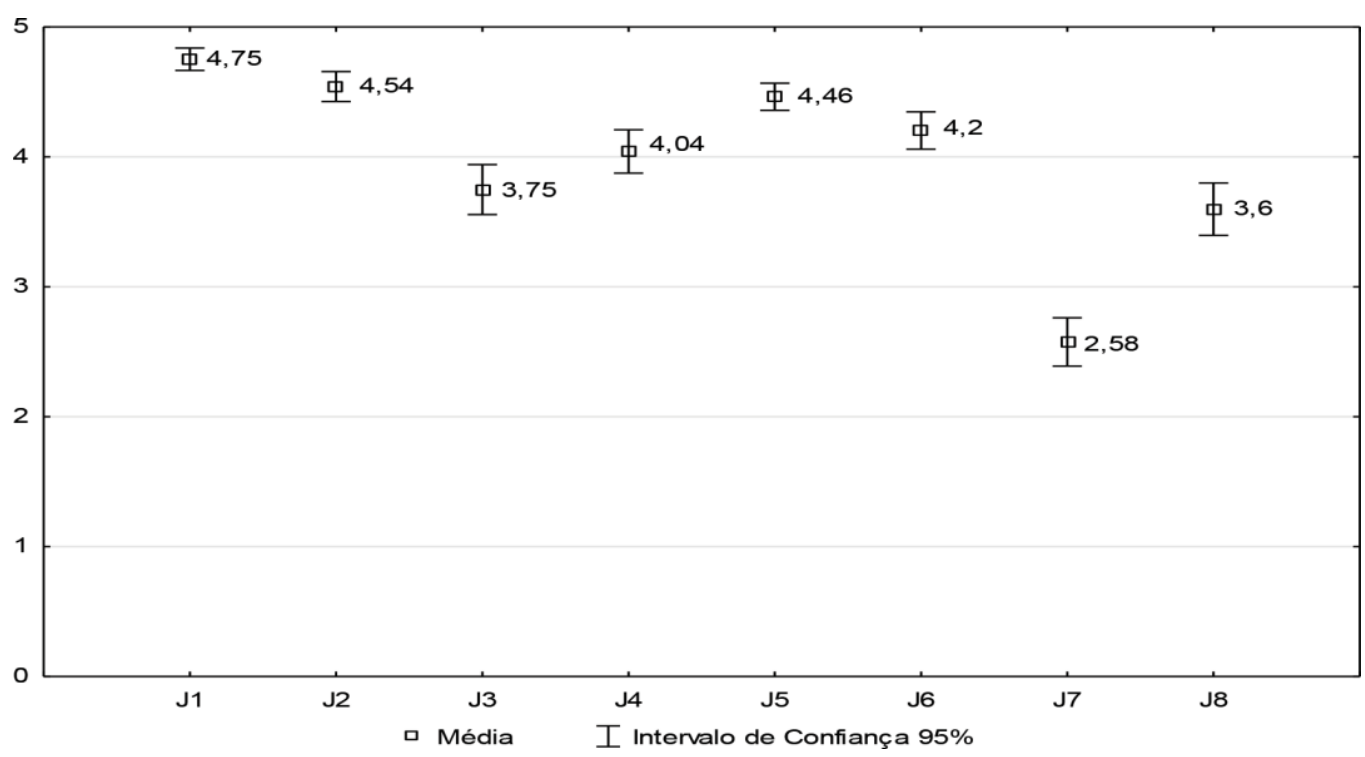

Legenda: J1 - tempo de atuação nas ações de controle da TB; J2 - abordagem do tema TB durante a formação; J3 - vivência da temática da TB em disciplinas ou estágios durante a formação; J4 - recebimento de treinamento específico no serviço para atuação em ações de controle da TB; J5 - qualificação dos profissionais para o atendimento de TB; J6 - qualificação dos profissionais para atuação segundo a diversidade cultural da comunidade; $\mathbf{J}$ - realização de educação permanente para o desenvolvimento de ações de controle da TB; J8 - seguimento de algum protocolo específico para as ações de controle da TB na unidade de saúde

Fonte: elaborado pelas autoras 
Dentre os aspectos que diferenciam a categorização geral, identificou-se a percepção inferior (quase sempre) de enfermeiros e ACS quanto à abordagem do tema TB durante a sua formação. Médicos apresentaram mais (sempre) disciplinas ou estágios que permitiram vivenciar a temática e treinamento específico no serviço para atuação em ações de controle da TB comparados com os demais profissionais, em especial, com os ACS e técnicos/auxiliares de enfermagem, que somente às vezes apresentaram a mesma vivência durante a sua formação e receberam treinamento específico no serviço, respectivamente (Figura 4).

Os ACS consideraram serem mais (sempre) qualificados para atender TB e trabalhar segundo a diversidade cultural da comunidade que os demais profissionais, ao passo que técnicos/auxiliares de enfermagem em menor peso (quase nunca) realizam educação permanente para o desenvolvimento de ações de controle da TB. Médicos e enfermeiros seguem mais (sempre) protocolos específicos para as ações de controle da TB na unidade de saúde do que técnicos/auxiliares de enfermagem, e ACS, que seguem meramente às vezes (Figura 4).

Figura 4-Representação gráfica da média e do intervalo de confiança dos escores da dimensão acesso ao tratamento, obtidos pelos profissionais enfermeiros (a), médicos (b), técnicos/auxiliares de enfermagem (c) e Agentes Comunitários de Saúde (d) entrevistados da APS em Porto Velho-RO, no período de maio de 2018 a fevereiro de 2019

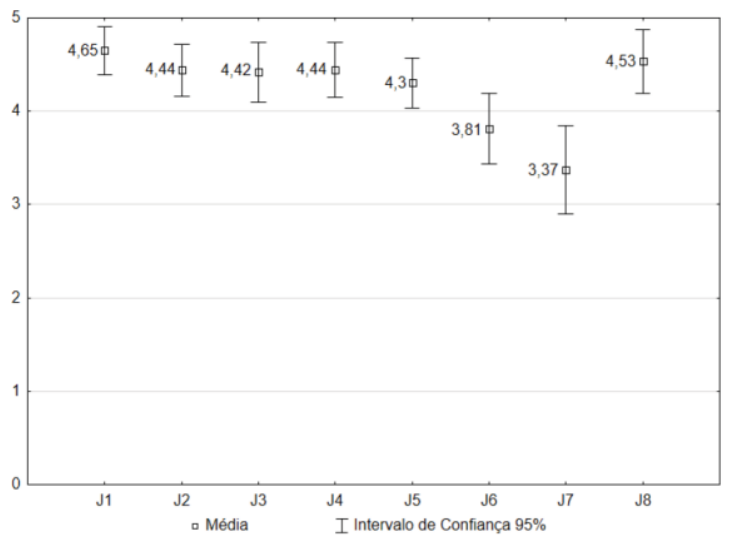

(a)

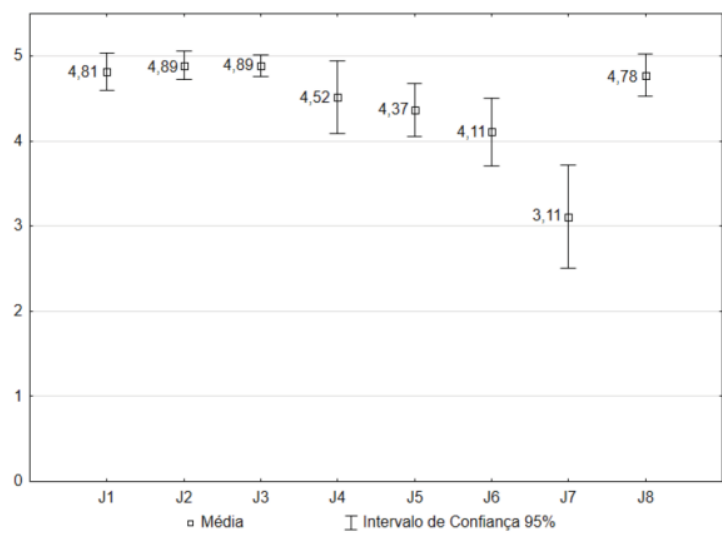

(b) 
(Conclusão)

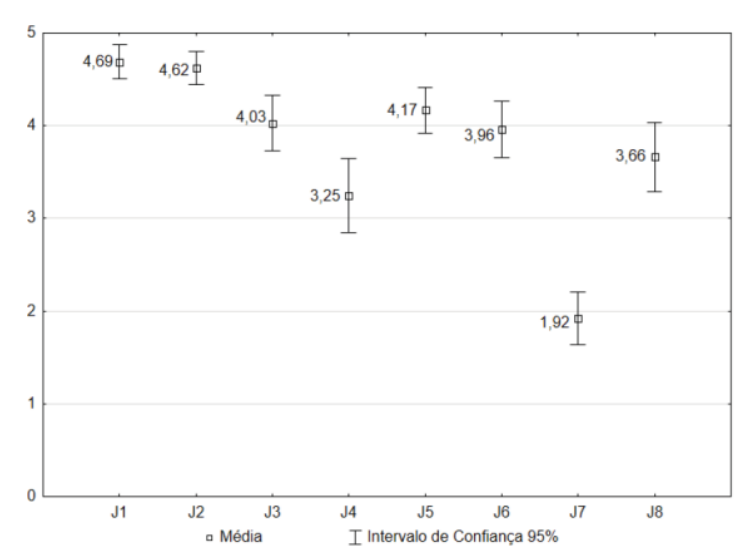

(c)

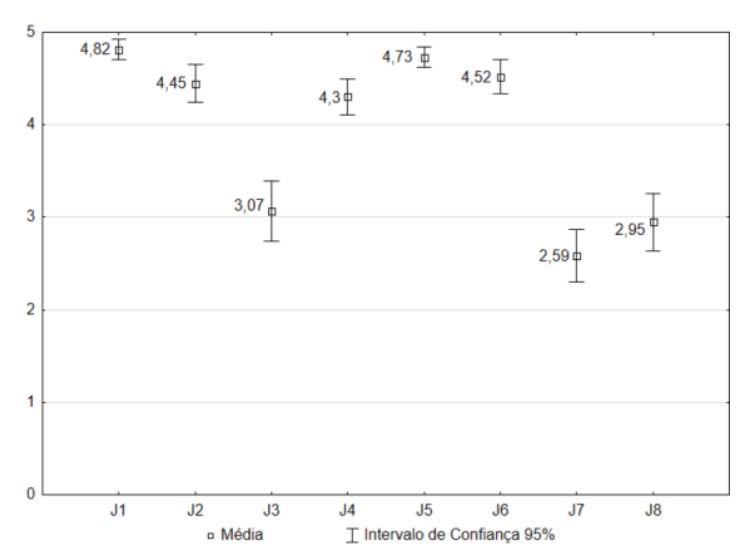

(d)

Legenda: J1 - tempo de atuação nas ações de controle da TB; J2 - abordagem do tema TB durante a formação; J3 - vivência da temática da TB em disciplinas ou estágios durante a formação; J4 - recebimento de treinamento específico no serviço para atuação em ações de controle da TB; J5 - qualificação dos profissionais para o atendimento de TB; J6 - qualificação dos profissionais para atuação segundo a diversidade cultural da comunidade; $\mathbf{J}$ - realização de educação permanente para o desenvolvimento de ações de controle da TB; J8 - seguimento de algum protocolo específico para as ações de controle da TB na unidade de saúde

$$
\text { Fonte: elaborado pelas autoras }
$$

\section{DISCUSSÃO}

A partir dos resultados encontrados, observa-se a disponibilidade do profissional médico para atendimento ao doente em tratamento, caso esse passe mal por causa da medicação ou da doença, entretanto, não garante o acesso à consulta. Haja vista as três dimensões que perpassam o conceito de acesso, ou seja, geográfica, organizacional e social ${ }^{3}$, considerando a distância entre o usuário e o serviço, a necessidade de transporte, que envolve ainda as condições socioeconômicas do doente, a forma como o acolhimento é ofertado (ou não) e o tempo gasto para se alcançar assistência 4 .

Quanto às atividades externas a unidade de saúde, as quais são fundamentais para a vigilância do espaço onde ocorre o adoecimento, os profissionais afirmaram quase sempre realizar visitas domiciliares aos doentes. ACS, no entanto, apresentaram classificação superior (sempre), divergindo de médicos e enfermeiros, que por sua vez apresentaram classificação inferior (às vezes) sobre a frequência das visitas domiciliares aos doentes de TB quando comparados com técnicos/auxiliares de enfermagem.

Observa-se, então, que tal assistência ocorre de forma isolada pelos ACS e, por isso, não vem sendo utilizada como ferramenta para o conhecimento das condições de vida das pessoas que moram na área de sua responsabilidade sanitária, como, por exemplo, emprego, moradia, saneamento básico. 
Tal fator implica em dificuldade de compreensão de que essas características podem ser barreiras enfrentadas pelos indivíduos, o que é evidenciado pela fragmentação da assistência, com responsabilização do ACS pelo acompanhamento domiciliar do doente de TB e pelas limitações que os servidores/serviços impõem acerca da visita domiciliar, como atividade exclusiva a pacientes idosos, acamados ou com dificuldades físicas de locomoção, conforme os relatos.

De forma global, os profissionais entendem que os doentes quase sempre pertencerem à área adscrita à unidade onde são tratados, diferenciando-se médicos e enfermeiros, com um entendimento inferior (às vezes) acerca do pertencimento à área, quando comparados com técnicos/auxiliares de enfermagem e ACS. Fato que está relacionado ao atendimento de doentes, especialmente para diagnóstico, de diferentes bairros do município.

Importante olhar que o município possui unidades de saúde com extensas áreas territoriais que distanciam do domicílio dos doentes de TB, tornando-se necessário que os mesmos utilizem algum tipo de transporte motorizado para se deslocar até o serviço de saúde, bem como arcar com o seu custo. Tal aspecto pode representar um obstáculo para o desenvolvimento do vínculo, desenvolvimento de ações como o TDO e adesão à terapia medicamentosa ${ }^{20,21}$, indo de encontro à afirmação de que o doente quase nunca tem dificuldade para se deslocar até a unidade onde faz tratamento.

Estudo comparativo do acesso geográfico em três cidades do Nordeste (Salvador, Recife e João pessoa) apontou que a cidade que apresentou menor mediana de distância percorrida pelos casos de TB até a unidade de saúde de atendimento obteve menor percentual de acesso dificultado. Esse fator esteve relacionado também a menor atendimento dos casos nos níveis secundários e terciários de atenção ${ }^{22}$ devido à maior aproximação com as unidades da APS.

Outra barreira de acesso ao serviço de saúde, no tocante à restrição do horário de atendimento e às fragilidades no acolhimento ${ }^{23}$, é observada quando os profissionais relatam que, às vezes, os doentes perdem turno de trabalho ou compromisso para serem atendidos, fato que é observado de modo inferior (quase nunca) pela equipe de enfermagem e ACS. Essa divergência com o profissional médico está relacionada à necessidade de atestado, o que ainda assim contradiz a afirmação de que os doentes de TB quase nunca esperam mais de 60 minutos.

Deve-se considerar que horários incompatíveis, demora no atendimento, atrasos e/ou faltas no trabalho para o comparecimento em consultas (mesmo que atestadas) podem distanciar os doentes dos serviços por receio de perder o emprego ${ }^{21}$.

A literatura aponta que a maioria dos doentes de TB teve perda de emprego ou da oportunidade de trabalhar como um dos principais problemas durante o tratamento. Os estudos relataram, ainda, que os doentes perderam o emprego quando o diagnóstico foi conhecido, não conseguiam continuar trabalhando por estarem muito afetados pelo 
adoecimento e/ou não conseguiam encontrar o trabalho por causa do tempo gasto com o tratamento ${ }^{4}, 21$.

Gebreweld e colaboradores ${ }^{21}$ abordam que o afastamento compulsório das atividades laborais leva ao comprometimento da renda familiar e, consequentemente, do tratamento, já que a condição socioeconômica leva a uma diminuição na qualidade nutricional da alimentação do doente, especialmente de alimentos ricos em proteína, como carne, leite e ovo, em razão do seu custo. Tal contexto aumenta o estigma social da TB, dificulta o diagnóstico precoce dos casos suspeitos e favorece outros desfechos, tais como o abandono e o óbito ${ }^{24}$.

Segundo as falas dos profissionais, os doentes não esperam em razão da transmissibilidade da doença, o que demonstra um desconhecimento quanto à TB. $\mathrm{O}$ uso da máscara independentemente da fase de tratamento foi outro discurso incoerente, além do receio que os doentes possuem de serem identificados perante a família e a comunidade. Tais aspectos fortalecem o estigma dentro e fora do serviço de saúde, o qual pode ser minimizado por meio de ações educativas ${ }^{25}$.

Ressalta-se sobre a importância do apoio familiar e comunitário para adesão ao tratamento, porque constitui uma rede de apoio no processo saúde-doença, auxilia a avaliação dos contatos, vigilância do espaço e compensa, inclusive, a perda de renda ${ }^{4,20,21}$.

O oferecimento de subsídios como cestas básicas e vale-transporte pode ser fundamental, mas exige dos serviços de saúde maior articulação intersetorial ${ }^{4}$. De modo complementar, a interação entre os níveis de atenção é fundamental até mesmo para a oferta da medicação, gratuita pelo SUS. Segundo os profissionais, quase nunca houve falta, mas como a disponibilidade desse insumo está relacionada à notificação e acompanhamento dos casos em tratamento, quando ocorre, pode estar relacionada às fragilidades desde o registro até o planejamento e organização das ações desenvolvidas pela equipe de saúde ${ }^{5}$.

O fato de quase nunca os doentes de TB conseguirem obter informações por telefone na unidade de saúde e nunca conseguirem marcar consulta por telefone tem relação com a disponibilidade deste nos serviços e profissionais, segundo o relato dos entrevistados. Entretanto, também são fatores que se configuram como obstáculo para o tratamento, ao se considerar que os meios de comunicação veem se tornando cada vez mais comuns e democráticos como ferramenta no manejo do cuidado e aproximação entre profissionais-unidades-usuários ${ }^{22}$, principalmente para as condições crônicas, como a TB.

Acerca da formação, os profissionais declararam que o tema TB sempre foi abordado, com menor proporção (quase sempre) para enfermeiros e ACS ao analisar por categoria profissional. Apesar da diferenciação, a frequência mantém-se elevada e vai de encontro à literatura, que indica que os mesmos apresentam fragilidades no conhecimento sobre os sinais, sintomas e transmissão da doença, ainda que 
apresentem maior tempo de serviço e treinamento para atuar nas ações de controle e vigilância da $\mathrm{TB}^{26}$.

Médicos apresentaram mais (sempre) disciplinas ou estágios que permitiram vivenciar a temática e treinamento específico no serviço para atuação em ações de controle da TB comparados com os demais profissionais. Do mesmo modo, ACS e técnicos/auxiliares de enfermagem, respectivamente, somente às vezes apresentaram a mesma vivência durante a sua formação e receberam treinamento específico no serviço, respectivamente.

Para todos, quase sempre tais vivências foram no âmbito hospitalar, favorecendo a execução de práticas curativistas, mecanicistas e fragmentadas, tendo como consequência a assistência voltada à clínica ${ }^{27}$, diferentemente do modelo de atenção preconizado na APS para a promoção da saúde e prevenção dos agravos.

Dentre os profissionais que compõe as eSF e eAB, enfermeiros e médicos, respectivamente, são os mais favorecidos pelos treinamentos teóricos e práticos sobre TB nos últimos anos, o que pode ser uma estratégia para viabilizar a descentralização da assistência à doença para APS $^{28}$. Considerando o contexto supracitado, faz-se necessário maior interação destes profissionais com os demais membros das equipes, com o objetivo de atuarem como multiplicadores do conhecimento adquirido e unificar os discursos e práticas.

A literatura aponta o protagonismo do enfermeiro nas ações de controle da TB, principalmente na gestão dos casos, desde o acompanhamento mensal dos casos

de TB, elaboração, registro e análise dos dados epidemiológicos, os quais são de responsabilidade da equipe de saúde, mas que fica alheia à assistência a doença $28,29,30,31$.

A coleta de dados apontou ainda uma assistência fragmentada, já que 95\% de todos os profissionais entrevistados já trabalhavam há mais de quatro anos em ações de controle da doença, mas $14,53 \%$ dos técnicos/auxiliares de enfermagem e 12,98\% dos ACS nunca haviam acompanhado nenhum caso de TB, apesar de os médicos e enfermeiros de suas equipes sempre terem vivenciado esse processo.

Chama a atenção, ainda, no discurso de alguns técnicos/auxiliares de enfermagem e ACS, a fala de que o questionário, ou parte dele, deveria ser respondido somente por médicos e enfermeiros, uma vez que os mesmos não tinham conhecimento/competência para tal, limitando-se (técnicos/auxiliares de enfermagem) aos procedimentos de enfermagem.

Todavia, ACS consideraram ser mais (sempre) qualificados para atender TB e trabalhar segundo a diversidade cultural da comunidade que os demais profissionais. Em oposição, técnicos/auxiliares de enfermagem realizam menos (quase nunca) educação permanente para o desenvolvimento de ações de controle da TB.

Como reflexo desse cenário, a responsabilização exclusiva do ACS pela busca ativa dos SR foi mais uma prática errônea identificada, uma vez que compete às atribuições 
da equipe multiprofissional ${ }^{32}$. Sabe-se também que essa ação exige saberes acerca da doença, como sinais e sintomas, modo de transmissão e orientação sobre a coleta de escarro. Entretanto, a menor possibilidade de participação em ações educativas em comparação com os profissionais de nível superior pode impactar na efetividade das ações desenvolvidas.

Muitos desses profissionais relataram não terem acompanhado caso de TB na área em que atuam, o que leva à reflexão do baixo percentual de cobertura da ESF no município e se os dados apresentados representam de fato o percentual coberto, de como vem ocorrendo a busca ativa dos casos e a priorização das visitas domiciliares, bem como a integração das eSF.

Diante dessas circunstâncias, ressalta-se a importância de capacitações que incluam toda a equipe e a da atuação de médicos e enfermeiros para educação permanente, já que somente às vezes os profissionais realizam ações educativas para o desenvolvimento de ações de controle da TB. Fator que se torna ainda mais preocupante ao observar o aspecto por categoria profissional, tendo em vista que técnicos/auxiliares de enfermagem quase nunca realizam ações nesse sentido, limitando-se a atividades mecanicista.

Essa necessidade é reforçada pelas falas de alguns profissionais que afirmaram ser necessário separar os utensílios domésticos dos doentes, deixando evidentes conceitos equivocados acerca do modo de transmissão da TB, e que são replicados durante a interação desses profissionais com a população.

A partir desses aspectos, questiona-se de que modo os profissionais quase sempre têm seguido algum protocolo específico para as ações de controle da doença na unidade de saúde, aspecto mais observado (sempre) com médicos e enfermeiros do que técnicos/auxiliares de enfermagem, e ACS, que seguem tão somente às vezes. E se os mesmos estão qualificados, de fato, para atender TB e atuar segundo a diversidade cultural da comunidade, conforme apontado por eles.

Em oposição às afirmações dos profissionais, as condições de formação impactam diretamente na atuação. Nesse sentido, o diagnóstico e o tratamento no nível terciário prejudicam a criação e fortalecimento de vínculo, que associados ao acesso e acolhimento pela equipe de saúde são fatores que podem (ou não) contribuir para o sucesso do tratamento, impactando no controle da doença enquanto problema de saúde pública e, consequentemente, na manutenção da cadeia de transmissão e resistência medicamentosa ${ }^{33}$.

\section{CONCLUSÃO}

O acesso ao tratamento da TB ocorre quando os fatores geográficos, organizacionais e sociais permitem que o usuário satisfaça as necessidades de saúde que perpassam o 
adoecimento. Por esse motivo, apenas a descentralização das ações de controle da TB para a APS não garante o alcance da assistência, sendo necessária a compreensão dos diversos fatores que agem como barreiras ao enfrentamento da doença.

Nesse sentido, a avaliação do desempenho da dimensão acesso ao tratamento apontou que apenas a disponibilidade para assistência não garante a sua concretização. Observou-se que as visitas domiciliares não são tidas como ferramenta para o conhecimento das condições sociais do doente, família e comunidade, o que reflete na dificuldade de compreensão dos profissionais de que esses aspectos podem representar barreiras a serem enfrentadas pelos usuários, as quais estão relacionadas também às condições dos serviços.

Quanto à formação profissional, apesar do tempo de serviço, do ensino acadêmico e da educação permanente, ainda se percebe assistência fragmentada e atenção centrada na clínica, assim como divergências entre as categorias profissionais, conforme o discurso apresentado, contrapondo a forma como os profissionais veem a assistência que prestam.

Portanto, torna-se fundamental ampliar a capacitação profissional a todos os membros da equipe, associando esse conhecimento ao contexto social, econômico e cultural da comunidade. O estudo limita-se por não avaliar o desempenho das dimensões em questão sob a ótica dos gestores e usuários, já que é notória uma discordância entre esses diferentes atores acerca do acesso, favorecendo mudanças no cenário local.

\section{REFERÊNCIAS}

1. Mendes EV. A construção social da atenção primária à saúde. Brasília: CONASS; 2015. $194 p$.

2. Starfield B. Atenção primária: equilíbrio entre necessidades de saúde, serviços e tecnologia. Brasília: UNESCO; 2002. 710 p.

3. Donabedian A. Aspects of Medical Care Administration. Boston: Haward; 1973. $800 \mathrm{p}$.

4. Furlan MCR, Marcon SS. Avaliação do acesso ao tratamento de tuberculose sob a perspectiva de usuários. Cad Saúde Colet. 2017; 25(3):339-47.

5. Ministério da Saúde (Brasil). Secretaria de Vigilância em Saúde. Departamento de Vigilância das Doenças Transmissíveis. Manual de Recomendações para o Controle da tuberculose no Brasil. Brasília: Ministério da Saúde; 2018.

6. Ministério da Saúde (Brasil). Secretaria de Vigilância em Saúde. Boletim Epidemiológico. Implantação do Plano Nacional pelo Fim da Tuberculose como Problema de Saúde Pública no Brasil: primeiros passos rumo ao alcance das metas. Brasília: Ministério da Saúde; 2018. 
7. Ministério da Saúde (Brasil). Secretaria de Vigilância em Saúde. Boletim Epidemiológico. Brasil Livre da Tuberculose: evolução dos cenários epidemiológicos e operacionais da doença. Brasília: Ministério da Saúde; 2019.

8. World Health Organization. The Stop TB Strategy. Geneva: WHO; 2006.

9. Silva LT, Felipini MCC, Oliveira TB, Brunello MEF, Orfão NH. Perfil epidemiológico da tuberculose no serviço de referência do estado de Rondônia. Rev. Epidemiol. Controle Infecç. 2019; 9(1).

10. Dye C, Glaziou P, Floyd K, Raviglione M. Prospects for Tuberculosis Elimination. Am J Epidemiol. 2013; 187(9):2011-20.

11. Ferreira MRL, Bonfim RO, Siqueira TC, Orfão NH. Abandono do tratamento da tuberculose: uma revisão integrativa. Rev Enferm Contemp. 2018; 7(1):63-71.

12. Siqueira TC, Bonfim RO, Ferreira MRL, Orfão NH. Mortalidade entre os portadores de tuberculose em Porto Velho (RO). Saúde e Pesqui. 2018; 11(3):441-50.

13. Rabahi MF, Silva Júnior JLR, Ferreira ACG, Tannus-Silva DGS, Conde MB. Tratamento da tuberculose. J Bras Pneumol. 2017; 43(5):472-86.

14. Santos MC, Andrade RPS, Macedo SM, Andrade ASS, Villa TCS, Pinto ESG. Organização da atenção primária para diagnóstico e tratamento da tuberculose. Cogitare Enferm. 2017; 22(2):e48151.

15. Cadastro Nacional de Estabelecimentos de Saúde [Internet]. Brasília: CNES. 2019 [citado em 2019 maio 5]. Disponível em: http://cnes.datasus.gov.br/pages/ estabelecimentos/consulta.jsp.

16. Informação e Gestão da Atenção Básica [Internet]: Brasília: EGESTORAB. 2019 - [citado em 2019 maio 28]. Disponível em: https://egestorab.saude.gov.br/index.xhtml.

17. Macinko J, Almeida C, Oliveira E. Avaliação das características organizacionais dos serviços de atenção básica em Petrópolis: teste de uma metodologia. Saud Debate. 2003; 27(65):243-56.

18. Villa TCS, Ruffino-Netto A. Tuberculose: pesquisas operacionais. Ribeirão Preto: FUNPEC; 2009. 249 p.

19. Brasil. Resolução 466, de 12 de dezembro de 2012. Brasília: Ministério da Saúde; 2012 [citado em 2019 fev 15]. Disponível em: http://bvsms.saude.gov.br/bvs/ saudelegis/cns/2013/res0466_12_12_2012.html.

20. Macedo SM, Andrade RPS, Souza CRBA, Andrade ASS, Villa TCS, Pinto ESG. Estratégias para Capacitação ao cuidado em Tuberculose. Cogitare Enferm. 2016; 21(3):1-8.

21. Gebreweld FH, Kifle MM, Gebremicheal FE, Simel LL, Gezae MM, Ghebreyesus SS, et al. Factors influencing adherence to tuberculosis treatment in Asmara, Eritrea: a qualitative study. J Health Popul Nutr. 2018;37(1):1-9. 
22. Souza MSPL, Aquino R, Pereira SM, Costa MCN, Barreto ML, Natividade, M, et al. Fatores associados ao acesso geográfico aos serviços de saúde por pessoas com tuberculose em três capitais do Nordeste brasileiro. Cad. Saúde Pública. 2015; 31(1):111-20.

23. Cardozo-Gonzales RI, Fredemir PP, Harter J, Alarcon E, Lima LML, Tomberg JO. Avaliação das ações de detecção de casos de tuberculose na atenção primária. Rev Eletr Enferm [Internet]. 2015 [acessado em 2018 dez 9]; 17(4):7. Disponível em: https://doi.org/10.5216/ree.v17i4.32846.

24. Loureiro RB, Villa TCS, Ruffino-Netto A, Peres RL, Braga JU, Zandonade E, et al. Acesso ao diagnóstico da tuberculose em serviços de saúde do município de Vitória, ES, Brasil. Ciênc. Saúde Coletiva. 2014; 19(4):1233-44.

25. Mabunda JT, Khoza LB, Borne HBV, Lebese RT. Needs assessment for adapting tb directly observed treatment intervention programme in Limpopo province, South Africa: A community-based participatory research approach. African J Prim Heal Care Fam Med. 2016; 8(2):1-7.

26. Silva-Sobrinho RA, Souza AL, Silva LMC, Wysocki AD, Beraldo AA, Villa TCS. Conhecimento de enfermeiros de unidades de atenção básica acerca da tuberculose. Cogitare Enferm. 2014; 19(1):34-40.

27. Campos RB, Silva-Sobrinho RA, Brunello MEF, Zilly A, Palha PF, Villa TCS. Controle da tuberculose em município fronteiriço: análise da capacidade institucional dos serviços de saúde. Cogitare Enferm. 2018; 23(2):e53251.

28. Silva-Sobrinho RA, Wysocki AD, Scatena LM, Pinto ESG, Beraldo AA, Andrade RLP, et al. Assessment of primary health care in the treatment of tuberculosis in a brazilian locality of the international triple frontier. Open Nurs J. 2017; 11:124-34.

29. Kebian LVA, Acioli S. A visita domiciliar de enfermeiros e agentes comunitários de saúde da Estratégia Saúde da Família. Rev. Eletr. Enf. 2014; 16(1):161-169.

30. Rêgo CCD, Macêdo SM, Andrade CRB, Maia VF, Pinto JTJM, Pinto ESG. Processo de trabalho do enfermeiro a pessoa com tuberculose na atenção primária. Rev Baiana Enferm. 2015; 29(3):218-28.

31. Wysocki AD, Ponce MAZ, Brunello MEF, Beraldo AA, Vendramini SHF, Scatena LM, Ruffino-Netto A, Villa TCS. Atenção Primária à Saúde e tuberculose: avaliação dos serviços. Rev Bras Epidemiol. 2017; 20 (1):161-75.

32. Brasil. Portaria $n^{\circ} 2.436$, de 21 de setembro de 2017. Brasília: Ministério da Saúde; 2017 [citado em 2019 fev 15]. Disponível em: http://bvsms.saude.gov.br/bvs/ saudelegis/gm/2017/prt2436_22_09_2017.html.

33. Neves RR, Ferro PS, Nogueira LMV, Rodrigues ILA. Acesso e vínculo ao tratamento de tuberculose na atenção primária em saúde. Rev Fund Care Online. 2016; 8(4):5143-49.

Submissão: agosto de 2019.

Aprovação: agosto de 2020. 